\title{
Pitfalls and differential diagnosis on adrenal lesions: current concepts in CT/MR imaging: a narrative review
}

\author{
Alfonso Reginelli ${ }^{1}$, Giovanna Vacca ${ }^{1}$, Mariapaola Belfiore ${ }^{1}$, Angelo Sangiovanni ${ }^{1}$, Valerio Nardone ${ }^{2}$, \\ Angelo Vanzulli ${ }^{3}$, Roberto Grassi ${ }^{1}$, Salvatore Cappabianca ${ }^{1}$ \\ ${ }^{1}$ Department of Precision Medicine, University of Campania “L. Vanvitelli”, Naples, Italy; ${ }^{2}$ Unit of Radiation Oncology, Ospedale del Mare, Naples, \\ Italy; ${ }^{3}$ Department of Radiology, University "La Statale" of Milan, Milan, Italy \\ Contributions: (I) Conception and design: A Reginelli; (II) Administrative support: None; (III) Provision of study materials or patients: G Vacca; (IV) \\ Collection and assembly of data: G Vacca; (V) Data analysis and interpretation: G Vacca; (VI) Manuscript writing: All authors; (VII) Final approval of \\ manuscript: All authors. \\ Correspondence to: Alfonso Reginelli. Department of Precision Medicine, University of Campania “L. Vanvitelli”, Piazza Miraglia, 2 80138, Napoli \\ (NA), Italy. Email: alfonsoreginelli@hotmail.com
}

\begin{abstract}
The purpose of this pictorial essay is to review the imaging findings of adrenal lesions. Adrenal lesions could be divided into functioning or non-functioning masses, primary or metastatic, and benign or malignant. Imaging techniques have undergone significant advances in recent years. The most significant objective of adrenal imaging is represented by the detection and, when possible, characterization of adrenal lesions in order to direct patient management correctly. The detection and management of adrenal lesions is based on cross-sectional imaging obtained with non-contrast CT (tumour density), contrast-enhanced CT including delayed washout (either absolute percentage washout or relative percentage one) and finally with MR chemical shift analysis (loss of signal intensity between in-phase and out-of-phase images including both qualitative and quantitative estimates of signal loss). The small incidental adrenal nodules are benign, in most of cases; some tumors such as lipid-rich adenoma and myelolipoma have characteristic features that can be diagnosed accurately in CT. On contrary, if the presenting contrast-enhanced CT shows an adrenal mass with uncertain or malignant morphologic features, particularly in patients with a known history of malignancy, further evaluations should be considered. The most significative implications for radiologists are represented by how to assess risk of malignancy on imaging and what follow-up to indicate if an adrenal incidentaloma is not surgically removed.
\end{abstract}

Keywords: Adrenal gland; computed tomography; magnetic resonance imaging; adrenal masses diagnosis

Submitted Jun 14, 2020. Accepted for publication Nov 11, 2020.

doi: $10.21037 /$ gs-20-559

View this article at: http://dx.doi.org/10.21037/gs-20-559

\section{Introduction}

The adrenal gland is a retroperitoneal bilateral organ located in the suprarenal area, with medial and lateral limbs looking like an inverted $\mathrm{Y}, \mathrm{V}$ or $\mathrm{L}$ in shape $(1,2)$. Despite of their position, adrenal lesions are frequently detected nowadays by radiological examinations thanks to the development of new diagnostic modalities such as ultrasound examination (US), computed tomography (CT) and magnetic resonance imaging (MR) and their widespread use (3-5). It is often possible to discover incidentally adrenal masses, the so called adrenal incidentaloma (6-8).

Incidental adrenal lesions are encountered in approximately $4 \%$ to $6 \%$ of the population on cross-sectional imaging; this rate increase to $7-10 \%$ of scans in older patients (9).

\footnotetext{
$\wedge$ ORCID: 0000-0003-4809-6235.
} 
Adrenal lesion may be observed also in emergency settings $(10,11)$ in which they can represent the principal emergency injury (hemorrhage, infarction or infection) or may be discovered in emergency settings incidentally in patient being investigated for non-specific acute abdominal pain (traumatic or not) (12-17).

The purpose of this narrative review is to describe CT and MR imaging features of adrenal lesions in order to distinguish first of all benign from malignant ones in order to direct patient management correctly. We present the following article in accordance with the NARRATIVE REVIEW reporting checklist (available at http://dx.doi. org/10.21037/gs-20-559).

\section{Imaging of adrenal lesions}

\section{Ultrasonography (US) imaging}

US is able to visualize adrenal lesions, but it is less sensitive and more operator dependent compared to computed tomography or magnetic resonance imaging (18-22). The examination of the right adrenal gland is possible in most of the cases. It seems to be more difficult to analyse the left gland because of the presence of the stomach and the smaller volume of the spleen (compared to the liver) (23).

Thanks to the lack of radiation, US should be considered as first step for the diagnosis of adrenal lesions and clinical management, when suspected, at least for children and pregnant women. Through US, it is possible principally to discriminate cystic mass respect to solid ones, but the definite and appropriate characterization of a mass remains difficult $(24,25)$.

\section{Computed tomography (CT) imaging}

CT is surely the most useful and widespread technique to examine the adrenal gland especially on the axial plane by sections of $\mathrm{f} 2.5-3 \mathrm{~mm}$ (26-30). Thanks to the presence of perinephric fat, CT can easily demonstrate location of lesions, their size and features especially when performed with the use of intravenous contrast agent.

When an adrenal mass is found incidentally on imaging, a dedicated CT protocol is usually performed to evaluate the mass. The overlapping venous phase enhancement patterns of adenomas and non-adenomas requires a delayed image series executed 10-15 minutes after contrast medium injection to establish if the lesion shows washout or not $(31,32)$. The absolute percentage washout (APW) and relative percentage washout (RPW) can be obtained through a set formula $(29,31,33)$.

\section{Magnetic resonance (MR) imaging}

MR should be considered especially if CT is not diagnostic. Various MR imaging parameters can be used in order to characterize adrenal masses, but the most useful is represented by the Chemical Shift Imaging (CSI) which allows the detection of intracellular lipid that is contained in most frequent adrenal lesions (adenomas) with loss of signal in the "out of phase" sequence (OP) (33-35). The loss of signal intensity can be demonstrated also with a quantitative analysis through specific formula based on the adrenal-to-spleen chemical shift ratio (ASR) or the adrenal sensitivity index (SII) $(31,36,37)$ avoiding liver and the muscle because of the frequent presence of fat content that may also cause signal loss on the OP sequences (31). 3T MRI system with the assessment of new sequences could allow to distinguish adenomas from non-adenoma but it has not contributed more than to the use of CSI $(31,38)$. MRI could be particularly useful to study adrenal lesions with an unenhanced CT attenuation between 10 and $30 \mathrm{HU}$, while contrast-enhanced CT might be more useful for analysing nodules with attenuation values greater than $30 \mathrm{HU}$ (39). The use of diffusion weighted imaging (DWI) is not useful to distinguish adenomas from non-adenomas (3). Some studies have considered dynamic gadolinium-enhanced MRI in order to distinguish metastases from adenomas; even if is not more sensitive than CSI for the characterization of adenoma, it is an important diagnostic tool for some adenomas that are not well characterized by CSI (3). A possibility to improve the diagnostic possibilities in cases of indeterminate and heterogeneous masses seems to be magnetic resonance spectroscopy (MRS) (40). Examining the choline-to-creatinine ratio, MRS may differentiate adenoma from non-adenoma but further studies will be needed (41).

\section{Benign adrenal conditions}

\section{Adenoma}

Adenoma is the most common benign adrenal tumor that arise from the cortex and it is formed by intra-cytoplasmatic fat. Incidence of adenomas increases with age and most of them are non-functioning (9). At unenhanced CT, adenomas are often incidentally discovered and they usually show 

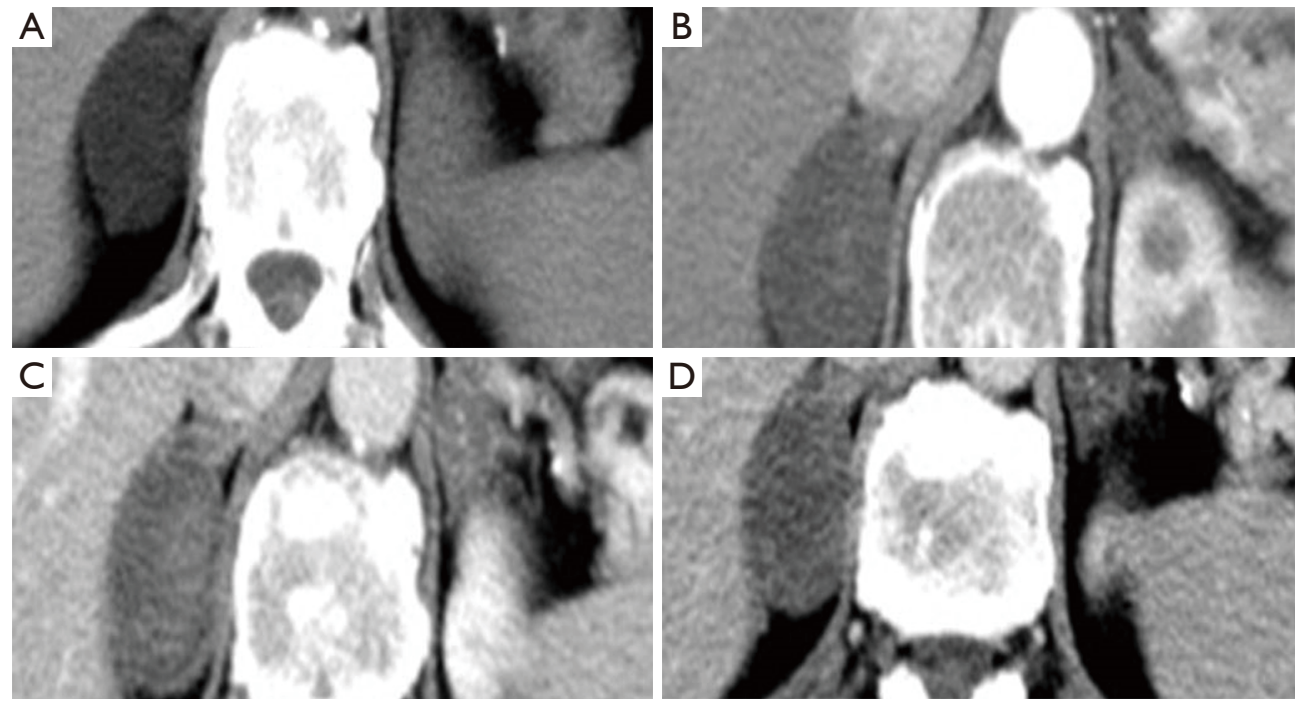

Figure 1 Unenhanced axial CT imaging show a voluminous right adrenal mass, with regular margins, and negative attenuation values (-4 $\mathrm{UH})$ (A). This lesion is characterized by rapid contrast enhancement during the arterial phase (68 UH) and also fast wash-out in portal phase $(18 \mathrm{UH})(\mathrm{B}, \mathrm{C}, \mathrm{D})$.
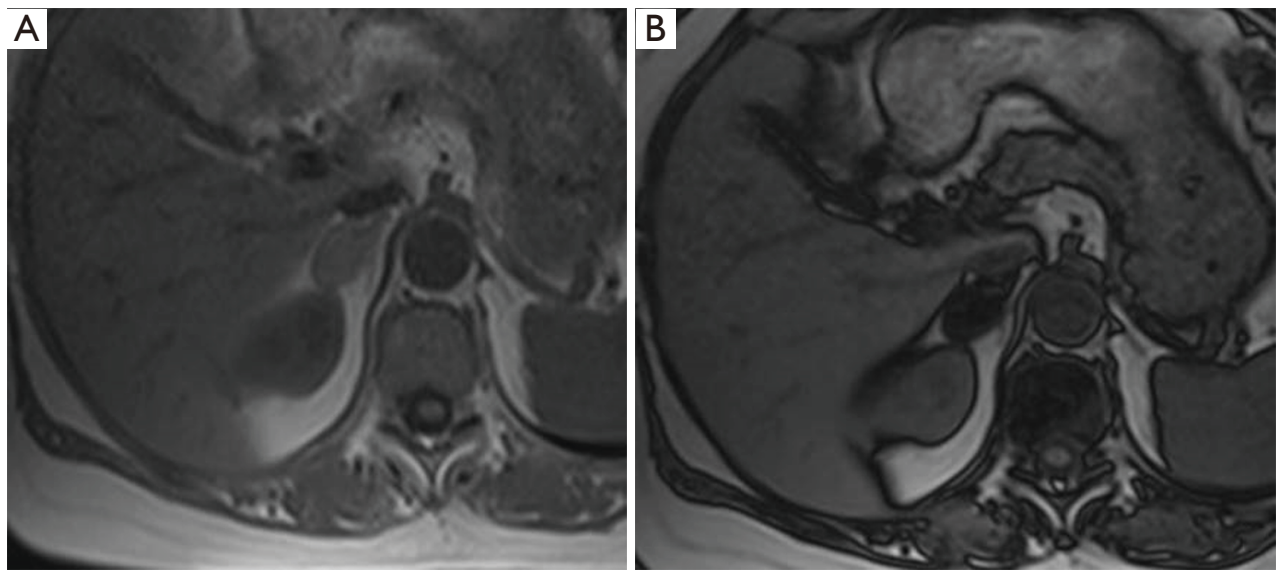

Figure 2 MR imaging of right adrenal adenoma (A). Chemical Shift Imaging (CSI) which allow the detection of intracellular lipid that is present in most frequent adrenal lesions (adenomas) (B).

round or oval shape, with homogeneous and attenuation values lower than 10 Hounsfield Units (HU) because of their high fat content (29) (Figure 1) even if $15-30 \%$ of them are characterized by higher attenuation values (39). In these cases, further and later acquisition phase should be added after 15 minutes from the intravenous contrast administration to differentiate assess diagnosis. Adenoma typically shows rapid and intense enhancement after contrast administration and a significant washout (APW $>60 \%$, RPW $>40 \%$ ) on delayed images both on CT or MR imaging (31). The loss of signal intensity on OP MRI is the most remarkable finding of adrenal adenoma (31) (Figure 2). Approximately $90 \%$ of adenomas presents a homogeneous or ring enhancement pattern on MRI and $60 \%$ of malignant lesions have a heterogeneous pattern (42) CSI has a sensitivity of $81-100 \%$ and specificity of $94-100 \%$, similar to those of non-contrast CT (41). No significant difference has been found between CT and MRI for lipidrich adenomas (43). CSI has been considered to be superior to non-contrast $\mathrm{CT}$ in the characterization of lipid-poor 
adenomas (43-45).

\section{Hemangioma}

Hemangioma are classified into 4 types: cavernous, venous, capillary type and mixed (46). On CT, hemangiomas can be homogeneous, even if in larger lesions is possible to discover calcifications which indicate previous hemorrhages (47). On contrast enhanced CT, hemangioma are often described with mainly peripheral enhancement, with or without centripetal pattern on delayed phases (47). At MR, hemangioma is hypointense on T1-weighted with a significant hyperintensity on T2-weighted images (47).

\section{Cyst}

Regarding the adrenal gland we have to distinguish pseudocysts (as a result from previous hemorrhage), endothelial cysts (deriving from lymphatic or arteriovenous malformations), epithelial cysts (with thin wall and serous liquid content), and parasitic cysts (39). On CT and MR, adrenal cysts generally show definite margins, homogeneous content (hypodense on CT and on MR hyperintense on T2weighted images); pseudocysts may have inhomogeneous content presenting a thicker cyst wall (47).

\section{Myelolipoma}

Myelolipoma is a benign tumor that is formed by fat and hematopoietic cells (39). At CT, myelolipoma looks like a well-demarked mass and its density depends on the proportion of fat and myeloid components. After contrast agent injection, myelolipoma typically shows low enhancement because of its poor vascularization (47). MR is fundamental for the diagnosis because the fat macroscopic components are hyperintense on $\mathrm{T} 1$ and $\mathrm{T} 2$-weighted images instead of the hematopoietic parts are hypointense on T1- and moderately hyperintense on T2-weighted images. Therefore the India ink artefact on CSI at the nodule-adrenal interface on opposed-phase images should suggest the diagnosis of myelolipoma (48).

\section{Pheocromocytoma}

Pheochromocytoma arises from the chromaffin cells of the adrenal medulla producing norepinephrine and epinephrine (49). Adrenal pheochromocytoma is commonly benign, although $10 \%$ of these lesions can be malignant (49). At CT, pheochromocytomas are very heterogeneous showing solid, cystic, calcific and necrotic components. After contrast administration, pheochromocytomas present high enhancement and their APW and RPW are similar to those of adenomas (50) so is often very difficult to differentiate pheochromocytomas from adenomas through $\mathrm{CT}$ adrenal protocol. MR is very sensitive for the diagnosis of pheochromocytomas (51) thanks to its hypointensity on T1-weighted and hyperintensity on $\mathrm{T} 2$-weighted images without loss of signal intensity on CSI, instead of the typical adenoma (39). The presence of somatostatin receptors makes possible the certain diagnosis of pheochromocytoma on PET imaging with ${ }^{68} \mathrm{Ga}$-DOTA-peptides (39).

\section{Lymphangioma}

Lymphangiomas are rare adrenal benign lesions with very non-specific features on contrast CT/MR imaging; therefore they appear on CT as hypodense lesion with intralesional septations or calcifications and on MR they show low signal intensity on T1 and high signal on T2weighted images (52).

\section{Schwannoma}

Schwannoma is a rare tumor arising from the sheath of the nerves (53); at CT, it appears as a well-circumscribed hypodense mass and at MR generally presents isointensity on T1-weighted images and slight hyperintensity on T2weighted images with heterogeneous contrast enhancement on both enhanced CT and MR imaging (53).

\section{Teratoma}

Teratoma of the adrenal gland is very uncommon (54). At CT it appears as a well-defined mass with cystic and fatty components and septations. Egg-shell calcification is a characteristic feature of an adrenal teratoma (54). On MRI, both T1- and T2-weighted images demonstrate the presence of many signals related to the different mass components. On enhanced CT and MRI, subtle enhancement of the solid part and internal septations with intense enhancement (54).

\section{Adrenal bematoma}

In adults, hematoma usually appears after abdominal 
trauma, especially when patients are under anticoagulant therapy (55). On CT the most specific feature of adrenal injury is a generalized enlargement of the gland with irregularity of adrenal shape (35) accompanied by high attenuation and regional fat inhomogeneity in up to $90 \%$ of cases (56-58).

MR is useful to detect the age of the adrenal hematoma. Diagnosis of adrenal hematoma could be difficult if it is caused by acute rupture of neoplasm (55). Acute intratumoral adrenal hemorrhage is seen in many conditions. Even if more commonly involves pheochromocytoma, hemorrhage has also been described in myelolipomas, metastatic lesions, adrenocortical carcinomas, adenomas, and hemangiomas and angiosarcoma (19). If there is an underlying tumor, morphologic imaging features could include intralesional calcification. Therefore, functional imaging through PET may show hypermetabolic activity and MR may be helpful for the detection of intralesional enhancement (55).

\section{Adrenal infarction}

Like other organs, it is possible to distinguish hemorrhagic and non hemorrhagic adrenal infarction. The first may lead to acute primary adrenal insufficiency and its own imaging features are similar to adrenal hematoma described above.

Non-hemorrhagic adrenal infarction is less common and it is often caused by thrombosis of the adrenal vein, which determine parenchymal necrosis (59). It may appear on CT as a hypoattenuating area that determine a diffuse enlargement of the adrenal gland, sometimes surrounded by a peripheral subtle line of hyperenhancement (the capsular sign) (60). It is frequent to notice stranding of fat and a small amount of fluid nearby the gland (60).

\section{Adrenal Infection}

Systemic viral infection may involve adrenal glands. Instead of vascular damage such as hematoma or infarction, adrenal infection may cause thickening of the gland surrounded by edema (61).

Cytomegalovirus and fungal agents such as Histoplasma capsulatum involve often bilaterally the adrenals determining adrenal insufficiency (62). Bacterial sepsis may determine bilateral adrenal hemorrhage and acute adrenal failure (the so-called Waterhouse-Friderichsen syndrome) (63). It is important to remember also that the adrenals could be the sites of Mycobacterium tuberculosis
(TB) infection showing enlargement of their volume and calcifications (64).

\section{Malignant adrenal conditions}

\section{Cortical carcinoma}

Cortical carcinoma is an uncommon malignant adrenal tumor that has higher incidence in childhood and middle age (65) and may be functioning especially in females; on CT, it presents malignant features like necrosis, calcifications and hemorrhage with heterogeneous and mainly peripheral enhancement (65). On MR, cortical carcinoma shows low signal intensity on T1-weighted images, high signal on T2-weighted images, and avid and irregular contrast enhancement with slow washout $(66,67)$. Adrenal carcinoma may infiltrate adjacent structures (kidney, inferior vena cava, splenic vessels) and disseminate to the liver, lung, and retroperitoneal lymph nodes (39).

\section{Lymphoma}

The primary adrenal lymphoma is very rare and in $50 \%$ of cases is a bilateral and generally associated with pathologic lymph adenopathy (68). Lymphoma lesions are typically large and homogeneous. On CT and MR imaging, adrenal secondary lymphomas are usually seen as soft tissue masses with slow and progressive enhancement (68).

\section{Metastases}

Metastases are the most frequent malignant adrenal lesions. The most common primary tumor that metastasizes to the adrenal glands is lung cancer followed by breast, colon, melanoma, kidney, and hepatocellular carcinoma (39). These malignant lesions are often bilateral and clinically silent; at CT, adrenal metastases present enhancement on the portal venous phase, but slower washout compared to adenomas (69) (Figure 3). At MR they generally appear hypointense on T1-weighted and moderately hyperintense on T2-weighted and they don't show signal loss on OP images (70). The differential diagnosis between metastasis and primary carcinoma is very difficult; carcinoma generally presents higher local invasiveness, especially on vascular structures (39). As a functional imaging modality, ${ }^{18} \mathrm{~F}-\mathrm{FDG}-\mathrm{PET} / \mathrm{CT}$ could be useful to distinguish indeterminate adrenal lesions when the suspect of malignancy is high (71). 

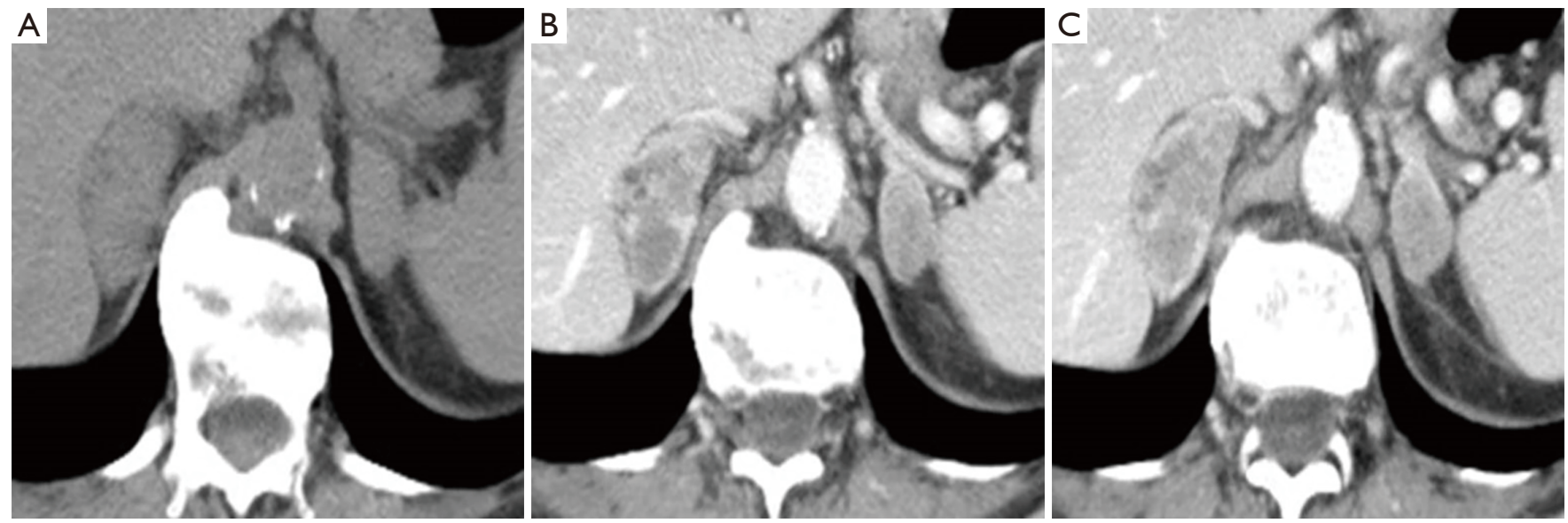

Figure 3 CT imaging show bilateral adrenal lesions (A). Morphological features such as size, bilateralism and content may lead to metastatic lesions in a patient affected by lung adenocarcinoma $(\mathrm{B}, \mathrm{C})$.

\section{Neuroblastoma}

Neuroblastoma is a neoplasm that arises from neural crest cells and is the second most common abdominal tumor in childhood after Wilms tumor (72). This lesion usually is heterogeneous showing calcification, necrosis, and intralesional hemorrhage (72). Generally, neuroblastoma becomes symptomatic when it invades or compresses adjacent structures (73). Neuroblastoma appears as a large heterogeneous lesion (73) on CT presenting intralesional calcifications that allows to distinguish this neoplasm from Wilms tumor (72,73). On MR neuroblastoma usually shows low signal on T1-weighted and high signal intensity on T2weighted images with heterogeneous contrast enhancement $(72,73)$.

\section{Methods}

We carefully searched in databases: PubMed/Medline, Web of Science, Google Scholar and Embase, from 2010 to 2020 to identify relevant studies, keywords including: "Adrenal gland" "computed tomography" "magnetic resonance imaging" "adrenal masses diagnosis". Eligible studies had to satisfy the following inclusion criteria: (I) recent studies (no longer than ten years ago); (II) English articles; (III) studies focused on clinical experience of using CT and MRI in the diagnosis and management of adrenal lesions.

\section{Discussion}

Adrenal lesions may be often discovered incidentally in patient being investigated for example for nonspecific acute abdominal pain (10). Radiologists must to be aware that a certain diagnosis is necessary for clinical management. For example if patients with benign adenomas can be clinically followed, patients with malignancies such as adrenocortical carcinoma or pheochromocytoma will require surgical evaluation and patients with a suspect of metastases should have first of all a thoracic evaluation, if not performed yet, because $50 \%$ of adrenal metastases are due to lung cancer (10).

Adrenal lesions could be divided into functioning or non-functioning masses, primary or metastatic, and benign or malignant.

CT contrast enhancement imaging is useful, especially for a non-hyperfunctioning mass, to differentiate benign from malignant adrenal lesions through two fundamental element:

- the intracellular lipid concentration of a mass;

- intravenous contrast pattern and washout behavior of a mass (27).

First of all, radiologists should keep in mind that the most frequent adrenal lesion who incidentally can be discovered on CT is represented by adrenal adenoma, even in oncologic patients $(74,75)$.

Unenhanced CT scan is sufficient to assess diagnosis of typical microscopic lipid rich adenoma (less than $10 \mathrm{HU}$ ) and myelolipoma with greater component of macroscopic fat and lower attenuation values $(-30 \mathrm{HU})(33)$.

Regarding lesions above $10 \mathrm{HU}$ on unenhanced CT is required contrast enhancement $\mathrm{CT}$ and/or MR imaging also with the study of the adrenal gland in the later phase 
(after 15 minutes from contrast intravenous injection) (76).

In this setting radiologist could discriminate adrenal benign or malignant lesion only through morphological imaging patterns like size, shape, margins and content (77).

Necrosis, calcifications and hemorrhage foci are suggestive for malignancy.

According to the American College of Radiology, if the size of the lesion is more than $4 \mathrm{~cm}$, or it increase more than $1 \mathrm{~cm}$ in 6 months, surgical resection might be considered only after an appropriate imaging study with adrenal specific cross-sectional imaging (76).

Regarding size, in a study conducted by Ctvrtlík et al., adenomas show significantly smaller size respect to malignant masses but differences in shape and margins of the tumor among the histological categories were not statistically significant (78).

Adenomas show more percentage of homogeneous structure in comparison with primary carcinomas, metastases, and pheochromocytomas $(79,80)$ instead of the presence of central hypodensity leading to necrosis that was found more frequently in primary carcinomas and pheochromocytomas.

Finally adenomas have faster washout rates respect to primary carcinomas, metastases, and pheochromocytomas (79-82).

These parameters are "suspicious imaging features", so not definitive indicators of malignancy and they can only contribute partially to the differentiation of adenomas from non-adenomas (83).

When we discover an adrenal incidentaloma, CT examination could be not sufficient to assess diagnosis without the study of the lesion's washout behaviour. Adenomas tend to de-enhance faster than non-adenomatous lesions because malignancies show vascular abnormalities and slow blood flow that allow the accumulation of contrast agents retaining it for a longer period (69).

Adrenal cysts are unilocular hypo-attenuated benign lesions with no enhancement and some of them show calcification of the wall without septations and blood products (84).

As we said before, acute hemorrhagic infarction should not be confused in this phase for an adrenal metastasis or rare postoperative abscess (85).

On the contrary when an organized adrenal hematoma show hypodense fluid central area is difficult to assess diagnosis and often a careful follow-up is necessary because only idiopathic hemorrhage typically decrease in volume (86).
According to us, the further clinical and diagnostic management of an adrenal incidentaloma is to differentiate patients with history of malignancy or not. In the first group of patients, less than 4-cm masses would be investigated with MR or CT adrenal specific examination without and with contrast.

In the second group of patients with no history of malignancy the evaluation of less than 4-cm masses can be executed only through dedicated CT or MR imaging repeating the examination in 12 months. If there are morphological changes during follow up, surgical resection should be considered. On contrary, if there is no mass change, no other further evaluation is needed (6).

Bilateral adrenal masses usually are benign (adenoma, macronodular hyperplasia) but metastases, lymphoma and pheocromocytomas should also be taken into account (87).

A new interesting clinical issue to consider is represented by the possible adverse reaction of some drugs which can involve adrenal glands. Ipilimumab, an anti-CTLA-4 receptor used for the treatment of many advanced malignant cancers such as melanoma or lung neoplasm could determine in most of cases hypophysitis but more rarely thyroid diseases or primary adrenal insufficiency $(88,89)$.

Particular settings involve also pregnant women and children.

Pregnancy has to be considerate as a para-physiologic state of hypercoagulability so the adrenal glands should be carefully investigated on MR in order to avoid ionized radiations if there is a clinical suspect of adrenal involvement (90). Adrenal infarcts could determine a diffuse hypointense enlargement of the adrenal gland with adjacent fluid/edema on T2-weighted images (90-93).

Adrenal hemorrhage are more common in children especially in neonates; in new-borns is more frequent compare to adults $(18,94-96)$ because adrenal glands seem to be more commonly involved traumatically because of the larger size and the hypervascularization in the neonatal period.

\section{Conclusions}

The most important aim of adrenal imaging is represented by the detection and, when possible, characterization of adrenal lesions in order to direct patient management correctly. In conclusion, when an adrenal mass shows malignant morphologic features at presenting contrastenhanced CT, this first examination would be not sufficient 
for mass characterization particularly in patients with a known history of malignancy (83). It should be necessary therefore to perform either dedicated contrast-enhanced adrenal CT (with a phase 15 min later than contrast medium injection) or MR examination with CSI (3).

The most significative implications for radiologists are nowadays represented by how to assess risk of malignancy on imaging, choosing the best second-stage imaging study to characterize indeterminate adrenal masses and what follow-up to indicate especially for adrenal incidentaloma (97-99).

\section{Acknowledgments}

Thanks to Valere Program by University of Campania "L. Vanvitelli".

Funding: None.

\section{Footnote}

Provenance and Peer Review: This article was commissioned by the Guest Editor (Antonio Barile) for the series "Multimodality Advanced Imaging and Intervention in Gland Diseases" published in Gland Surgery. The article has undergone external peer review.

Reporting Checklist: The authors have completed the NARRATIVE REVIEW reporting checklist. Available at http://dx.doi.org/10.21037/gs-20-559

Conflicts of Interest: All authors have completed the ICMJE uniform disclosure form (available at http://dx.doi. org/10.21037/gs-20-559). The series "Multimodality Advanced Imaging and Intervention in Gland Diseases" was commissioned by the editorial office without any funding or sponsorship. The authors have no other conflicts of interest to declare.

Ethical Statement: The authors are accountable for all aspects of the work in ensuring that questions related to the accuracy or integrity of any part of the work are appropriately investigated and resolved.

Open Access Statement: This is an Open Access article distributed in accordance with the Creative Commons Attribution-NonCommercial-NoDerivs 4.0 International License (CC BY-NC-ND 4.0), which permits the noncommercial replication and distribution of the article with the strict proviso that no changes or edits are made and the original work is properly cited (including links to both the formal publication through the relevant DOI and the license). See: https://creativecommons.org/licenses/by-nc-nd/4.0/.

\section{References}

1. Malayeri AA, Zaheer A, Fishman EK, et al. Adrenal masses: contemporary imaging characterization. J Comput Assist Tomogr 2013;37:528-42.

2. Mihai R. Surgical anatomy of the adrenal gland. Gland Surg 2019;8:S1-S2.

3. d'Amuri FV, Maestroni U, Pagnini F, et al. Magnetic resonance imaging of adrenal gland: state of the art. Gland Surg 2019;8:S223-32.

4. Reginelli A, Vacca G, Zanaletti N, et al. Diagnostic value/performance of radiological liver imaging during chemoterapy for gastrointestinal malignancy: A critical review. Acta Biomedica 2019;90:51-61.

5. Caranci F, Brunese L, Reginelli A, et al. Neck neoplastic conditions in the emergency setting: role of multidetector computed tomography. Semin Ultrasound CT MR 2012;33:443-8.

6. Willatt JM, Francis IR. Radiologic evaluation of incidentally discovered adrenal masses. Am Fam Physician 2010;81:1361-6.

7. Schieda N, Siegelman ES. Update on CT and MRI of Adrenal Nodules. AJR Am J Roentgenol 2017;208:1206-17.

8. Reginelli A, Di Grezia G, Izzo A, et al. Imaging of adrenal incidentaloma: our experience. Int J Surg 2014;12 Suppl 1:S126-31.

9. Blake MA, Holalkere NS, Boland GW. Imaging techniques for adrenal lesion characterization. Radiol Clin North Am 2008;46:65-78, vi.

10. Chernyak V, Patlas MN, Menias CO, et al. Traumatic and non-traumatic adrenal emergencies. Emerg Radiol 2015;22:697-704.

11. Addeo G, Cozzi D, Danti G, et al. Multi-detector computed tomography in the diagnosis and characterization of adrenal gland traumatic injuries. Gland Surg 2019;8:164-73.

12. Reginelli A, Zappia M, Barile A, et al. Strategies of imaging after orthopedic surgery. Musculoskelet Surg 2017;101:1.

13. Bruno F, Arrigoni F, Maggialetti N, et al. Neuroimaging in emergency: a review of possible role of pineal gland disease. Gland Surg 2019;8:133-40. 
14. Pinto A, Reginelli A, Pinto F, et al. Errors in imaging patients in the emergency setting. Br J Radiol 2016;89:20150914.

15. Ierardi AM, Xhepa G, Duka E, et al. Ethylene-vinyl alcohol polymer trans-arterial embolization in emergency peripheral active bleeding: initial experience. Int Angiol 2015;34:28-35.

16. Reginelli A, Pinto A, Russo A, et al. Sharp penetrating wounds: spectrum of imaging findings and legal aspects in the emergency setting. Radiol Med 2015;120:856-65.

17. Barile A, Brunese L, Giovagnoni A. Gland diseases: new perspectives in diagnostic radiology. Gland Surg 2019;8:S126-9.

18. Chira RI, Chira A, Manzat-Saplacan RM, et al. Adrenal glands transabdominal ultrasonography - pictorial essay. Med Ultrason 2017;19:318-23.

19. Suzuki Y, Sasagawa, Suzuki H, et al. The role of ultrasonography in the detection of adrenal masses: comparison with computed tomography and magnetic resonance imaging. Int Urol Nephrol 2001;32:303-6.

20. Ahn HS, Lee JB, Seo M, et al. Distinguishing benign from malignant thyroid nodules using thyroid ultrasonography: utility of adding superb microvascular imaging and elastography. Radiol Med 2018;123:260-70.

21. Scialpi M, Reginelli A, D'Andrea A, et al. Pancreatic tumors imaging: An update. Int J Surg 2016;28 Suppl $1: S 142-55$.

22. Reginelli A, Urraro F, di Grezia G, et al. Conventional ultrasound integrated with elastosonography and B-flow imaging in the diagnosis of thyroid nodular lesions. Int J Surg 2014;12 Suppl 1:S117-22.

23. Dietrich CF, Wehrmann T, Hoffmann C, et al. Detection of the adrenal glands by endoscopic or transabdominal ultrasound. Endoscopy 1997;29:859-64.

24. Tagliati C, Argalia G, Graziani B, et al. Contrast-enhanced ultrasound in the evaluation of splenic injury healing time and grade. Radiol Med 2019;124:163-9.

25. Tagliati C, Argalia G, Polonara G, et al. Contrastenhanced ultrasound in delayed splenic vascular injury and active extravasation diagnosis. Radiol Med 2019;124:170-5.

26. Ozturk E, Onur Sildiroglu H, Kantarci M, et al. Computed tomography findings in diseases of the adrenal gland. Wien Klin Wochenschr 2009;121:372-81.

27. Blake MA, Kalra MK, Sweeney AT, et al. Distinguishing benign from malignant adrenal masses: multi-detector row CT protocol with 10-minute delay. Radiology 2006;238:578-85.
28. Slattery JM, Blake MA, Kalra MK, et al. Adrenocortical carcinoma: contrast washout characteristics on CT. AJR Am J Roentgenol 2006;187:W21-4.

29. Caoili EM, Korobkin M, Francis IR, et al. Adrenal masses: characterization with combined unenhanced and delayed enhanced CT. Radiology 2002;222:629-33.

30. Agostini A, Borgheresi A, Mari A, et al. Dual-energy CT: theoretical principles and clinical applications. Radiol Med 2019;124:1281-95.

31. Guerrisi A, Marin D, Baski M, et al. Adrenal lesions: spectrum of imaging findings with emphasis on multidetector computed tomography and magnetic resonance imaging. J Clin Imaging Sci 2013;3:61.

32. Foti G, Malleo G, Faccioli N, et al. Characterization of adrenal lesions using MDCT wash-out parameters: diagnostic accuracy of several combinations of intermediate and delayed phases. Radiol Med 2018;123:833-40.

33. Korobkin M, Giordano TJ, Brodeur FJ, et al. Adrenal adenomas: relationship between histologic lipid and CT and MR findings. Radiology 1996;200:743-7.

34. Israel GM, Korobkin M, Wang C, et al. Comparison of unenhanced CT and chemical shift MRI in evaluating lipid-rich adrenal adenomas. AJR Am J Roentgenol 2004;183:215-9.

35. Mayo-Smith WW, Lee MJ, McNicholas MM, et al. Characterization of adrenal masses $(<5 \mathrm{~cm})$ by use of chemical shift MR imaging: observer performance versus quantitative measures. AJR Am J Roentgenol 1995;165:91-5.

36. Taffel M, Haji-Momenian S, Nikolaidis P, et al. Adrenal imaging: a comprehensive review. Radiol Clin North Am 2012;50:219-43, v.

37. Cappabianca S, Iaselli F, Reginelli A, et al. Value of diffusion-weighted magnetic resonance imaging in the characterization of complex adnexal masses. Tumori 2013;99:210-7.

38. Marin D, Dale BM, Bashir MR, et al. Effectiveness of a three-dimensional dual gradient echo two-point Dixon technique for the characterization of adrenal lesions at 3 Tesla. Eur Radiol 2012;22:259-68.

39. Albano D, Agnello F, Midiri F, et al. Imaging features of adrenal masses. Insights Imaging 2019;10:1.

40. Melo HJ, Goldman SM, Szejnfeld J, et al. Application of a protocol for magnetic resonance spectroscopy of adrenal glands: an experiment with over 100 cases. Radiol Bras 2014;47:333-41.

41. Blake MA, Cronin CG, Boland GW. Adrenal imaging. AJR Am J Roentgenol 2010;194:1450-60. 
42. Korivi BR, Elsayes KM. Cross-sectional imaging work-up of adrenal masses. World J Radiol 2013;5:88-97.

43. Haider MA, Ghai S, Jhaveri K, et al. Chemical shift MR imaging of hyperattenuating ( $>10 \mathrm{HU})$ adrenal masses: does it still have a role? Radiology 2004;231:711-6.

44. Vitale G, Tortora F, Baldelli R, et al. Pituitary magnetic resonance imaging in Cushing's disease. Endocrine 2017;55:691-6.

45. Tortora F, Prudente M, Cirillo M, et al. Diagnostic accuracy of short-time inversion recovery sequence in Graves' Ophthalmopathy before and after prednisone treatment. Neuroradiology 2014;56:353-61.

46. Sandrasegaran K, Patel AA, Ramaswamy R, et al. Characterization of adrenal masses with diffusion-weighted imaging. AJR Am J Roentgenol 2011;197:132-8.

47. Lattin GE, Jr., Sturgill ED, Tujo CA, et al. From the radiologic pathology archives: Adrenal tumors and tumor-like conditions in the adult: radiologic-pathologic correlation. Radiographics 2014;34:805-29.

48. Galia M, Albano D, Bruno A, et al. Imaging features of solid renal masses. Br J Radiol 2017;90:20170077.

49. Blake MA, Kalra MK, Maher MM, et al.

Pheochromocytoma: an imaging chameleon.

Radiographics 2004;24 Suppl 1:S87-99.

50. Patel J, Davenport MS, Cohan RH, et al. Can established CT attenuation and washout criteria for adrenal adenoma accurately exclude pheochromocytoma? AJR Am J Roentgenol 2013;201:122-7.

51. Jacques AE, Sahdev A, Sandrasagara M, et al. Adrenal phaeochromocytoma: correlation of MRI appearances with histology and function. Eur Radiol 2008;18:2885-92.

52. Rowe SP, Bishop JA, Prescott JD, et al. CT Appearance of Adrenal Cystic Lymphangioma: Radiologic-Pathologic Correlation. AJR Am J Roentgenol 2016;206:81-5.

53. Inokuchi T, Takiuchi H, Moriwaki Y, et al. Retroperitoneal ancient schwannoma presenting as an adrenal incidentaloma: CT and MR findings. Magn Reson Imaging 2006;24:1389-93.

54. Wang F, Liu J, Zhang R, et al. CT and MRI of adrenal gland pathologies. Quant Imaging Med Surg 2018;8:853-75.

55. Jordan E, Poder L, Courtier J, et al. Imaging of nontraumatic adrenal hemorrhage. AJR Am J Roentgenol 2012;199:W91-8.

56. Sinelnikov AO, Abujudeh HH, Chan D, et al. CT manifestations of adrenal trauma: experience with 73 cases.
Emerg Radiol 2007;13:313-8.

57. To'o KJ, Duddalwar VA. Imaging of traumatic adrenal injury. Emerg Radiol 2012;19:499-503.

58. Burks DW, Mirvis SE, Shanmuganathan K. Acute adrenal injury after blunt abdominal trauma: CT findings. AJR Am J Roentgenol 1992;158:503-7.

59. Fox B. Venous infarction of the adrenal glands. J Pathol 1976;119:65-89.

60. Moschetta M, Telegrafo M, Pignatelli A, et al. Value of the CT "capsular sign" as a potential indicator of acute adrenal ischemia. Emerg Radiol 2015;22:533-8.

61. Upadhyay J, Sudhindra P, Abraham G, et al. Tuberculosis of the adrenal gland: a case report and review of the literature of infections of the adrenal gland. Int J Endocrinol 2014;2014:876037.

62. Glasgow BJ, Steinsapir KD, Anders K, et al. Adrenal pathology in the acquired immune deficiency syndrome. Am J Clin Pathol 1985;84:594-7.

63. Joshi P, Lele V. FDG PET/CT findings in a case of nontuberculous abscess of adrenal gland. Clin Nucl Med 2014;39:57-8.

64. Lam KY, Lo CY. A critical examination of adrenal tuberculosis and a 28-year autopsy experience of active tuberculosis. Clin Endocrinol (Oxf) 2001;54:633-9.

65. Ng L, Libertino JM. Adrenocortical carcinoma: diagnosis, evaluation and treatment. J Urol 2003;169:5-11.

66. Bharwani N, Rockall AG, Sahdev A, et al. Adrenocortical carcinoma: the range of appearances on CT and MRI. AJR Am J Roentgenol 2011;196:W706-14.

67. Ferrozzi F, Bova D. CT and MR demonstration of fat within an adrenal cortical carcinoma. Abdom Imaging 1995;20:272-4.

68. Leite NP, Kased N, Hanna RF, et al. Cross-sectional imaging of extranodal involvement in abdominopelvic lymphoproliferative malignancies. Radiographics 2007;27:1613-34.

69. Mayo-Smith WW, Boland GW, Noto RB, et al. State-ofthe-art adrenal imaging. Radiographics 2001;21:995-1012.

70. Dong Y, Liu Q. Differentiation of malignant from benign pheochromocytomas with diffusion-weighted and dynamic contrast-enhanced magnetic resonance at 3.0 T. J Comput Assist Tomogr 2012;36:361-6.

71. Delivanis DA, Bancos I, Atwell TD, et al. Diagnostic performance of unenhanced computed tomography and (18) F-fluorodeoxyglucose positron emission tomography in indeterminate adrenal tumours. Clin Endocrinol (Oxf) 
2018;88:30-6.

72. Rha SE, Byun JY, Jung SE, et al. Neurogenic tumors in the abdomen: tumor types and imaging characteristics. Radiographics 2003;23:29-43.

73. Lonergan GJ, Schwab CM, Suarez ES, et al. Neuroblastoma, ganglioneuroblastoma, and ganglioneuroma: radiologic-pathologic correlation. Radiographics 2002;22:911-34.

74. Korobkin M, Francis IR, Kloos RT, et al. The incidental adrenal mass. Radiol Clin North Am 1996;34:1037-54.

75. Sheafor DH, Kovacs MD, Burchett $P$, et al. Impact of low-kVp scan technique on oral contrast density at abdominopelvic CT. Radiol Med 2018;123:918-25.

76. Choyke PL. ACR Appropriateness Criteria on incidentally discovered adrenal mass. J Am Coll Radiol 2006;3:498-504.

77. Johnson PT, Horton KM, Fishman EK. Adrenal imaging with multidetector CT: evidence-based protocol optimization and interpretative practice. Radiographics 2009;29:1319-31.

78. Ctvrtlík F, Herman M, Student V, et al. Differential diagnosis of incidentally detected adrenal masses revealed on routine abdominal CT. Eur J Radiol 2009;69:243-52.

79. Hamrahian AH, Ioachimescu AG, Remer EM, et al. Clinical utility of noncontrast computed tomography attenuation value (hounsfield units) to differentiate adrenal adenomas/hyperplasias from nonadenomas: Cleveland Clinic experience. J Clin Endocrinol Metab 2005;90:871-7.

80. Caoili EM, Korobkin M, Francis IR, et al. Delayed enhanced CT of lipid-poor adrenal adenomas. AJR Am J Roentgenol 2000;175:1411-5.

81. Szolar DH, Korobkin M, Reittner P, et al. Adrenocortical carcinomas and adrenal pheochromocytomas: mass and enhancement loss evaluation at delayed contrast-enhanced CT. Radiology 2005;234:479-85.

82. Korobkin M, Brodeur FJ, Francis IR, et al. CT timeattenuation washout curves of adrenal adenomas and nonadenomas. AJR Am J Roentgenol 1998;170:747-52.

83. Song JH, Grand DJ, Beland MD, et al. Morphologic features of 211 adrenal masses at initial contrast-enhanced CT: can we differentiate benign from malignant lesions using imaging features alone? AJR Am J Roentgenol 2013;201:1248-53.

84. Gross MD, Korobkin M, Bou Assaly W, et al. Contemporary imaging of incidentally discovered adrenal masses. Nat Rev Urol 2009;6:363-73.

85. Boraschi P, Donati F. Complications of orthotopic liver transplantation: imaging findings. Abdom Imaging 2004;29:189-202.

86. Kawashima A, Sandler CM, Ernst RD, et al. Imaging of nontraumatic hemorrhage of the adrenal gland. Radiographics 1999;19:949-63.

87. Sahdev A. Recommendations for the management of adrenal incidentalomas: what is pertinent for radiologists? Br J Radiol 2017;90:20160627.

88. Seki T, Yasuda A, Oki M, et al. Secondary Adrenal Insufficiency Following Nivolumab Therapy in a Patient with Metastatic Renal Cell Carcinoma. Tokai J Exp Clin Med 2017;42:115-20.

89. Nardone V, Tini P, Pastina P, et al. Radiomics predicts survival of patients with advanced non-small cell lung cancer undergoing PD-1 blockade using Nivolumab. Oncol Lett 2020;19:1559-66.

90. Ghasemi M, Beigi AA, Behnaz F, et al. Spontaneous Adrenal Hematoma in a Pregnant Woman; a Case Report. Emerg (Tehran) 2017;5:e59.

91. Tomà $\mathrm{P}$, Bartoloni $\mathrm{A}$, Salerno $\mathrm{S}$, et al. Protecting sensitive patient groups from imaging using ionizing radiation: effects during pregnancy, in fetal life and childhood. Radiol Med 2019;124:736-44.

92. Giurazza F, Corvino F, Silvestre M, et al. Adrenal glands hemorrhages: embolization in acute setting. Gland Surg 2019;8:115-22.

93. Ali A, Singh G, Balasubramanian SP. Acute non-traumatic adrenal haemorrhage-management, pathology and clinical outcomes. Gland Surg 2018;7:428-32.

94. Tomà $\mathrm{P}$, Esposito F. Up-to-date imaging review of paediatric soft tissue vascular masses, focusing on sonography. Radiol Med 2019;124:935-45.

95. Qin L, Ma Z, Yan F, et al. Iterative model reconstruction (IMR) algorithm for reduced radiation dose renal artery CT angiography with different tube voltage protocols. Radiol Med 2018;123:83-90.

96. Molinelli V, Iosca S, Duka E, et al. Ability of specific and nonspecific signs of multidetector computed tomography (MDCT) in the diagnosis of blunt surgically important bowel and mesenteric injuries. Radiol Med 2018;123:891-903.

97. Dinnes J, Bancos I, Ferrante di Ruffano L, et al. MANAGEMENT OF ENDOCRINE DISEASE: Imaging for the diagnosis of malignancy in incidentally 
discovered adrenal masses: a systematic review and metaanalysis. Eur J Endocrinol 2016;175:R51-64.

98. Makay O, Erol V, Ozdemir M. Robotic adrenalectomy. Gland Surg 2019;8:S10-6.

Cite this article as: Reginelli A, Vacca G, Belfiore M, Sangiovanni A, Nardone V, Vanzulli A, Grassi R, Cappabianca S. Pitfalls and differential diagnosis on adrenal lesions: current concepts in CT/MR imaging: a narrative review. Gland Surg 2020;9(6):2331-2342. doi: 10.21037/gs-20-559
99. Ierardi AM, Petrillo M, Patella F, et al. Interventional radiology of the adrenal glands: current status. Gland Surg 2018;7:147-65. 